\title{
MicroRNA-301a promotes cell proliferation via PTEN targeting in Ewing's sarcoma cells
}

\author{
MASANORI KAWANO, KAZUHIRO TANAKA, ICHIRO ITONAGA, \\ TATSUYA IWASAKI and HIROSHI TSUMURA
}

Department of Orthopaedic Surgery, Faculty of Medicine, Oita University, Oita 879-5593, Japan

Received December 6, 2015; Accepted January 13, 2016

DOI: $10.3892 /$ ijo.2016.3379

\begin{abstract}
MicroRNAs (miRNAs) regulate cell proliferation and differentiation by affecting gene expression at the posttranscriptional level by binding to complementary sequences within mRNAs in cancer cells, indicating that miRNAs can function as tumor suppressors or oncogenes. Recent studies showed that dysregulation of miRNA expression was associated with increased tumorigenicity and poor prognosis in several types of cancers, including Ewing's sarcoma (ES). To explore possible oncogenic factors in ES, we conducted microarray-based investigation and profiled the changes in miRNA expression and their effects on downstream mRNAs in five ES cell lines and human mesenchymal stem cells (hMSCs). miR-301a was significantly upregulated, while the phosphatase and tensin homolog (PTEN) expression was significantly downregulated in all tested ES cells as compared to hMSCs. When anti-miR-301a was transfected into ES cell lines, PTEN expression was significantly enhanced, suggesting that PTEN might be a target of miR-301a in ES cells. The expression of protein kinase $\mathrm{B}(\mathrm{Akt})$, which is inversely correlated with PTEN expression, was significantly downregulated in antimiR-301a-transfected cells. Additionally, the transfection of anti-miR-301a inhibited ES cell proliferation and cell cycle progression. Furthermore, downregulation of miR-301a in ES cells significantly suppressed tumor growth in vivo. Our results demonstrated the novel mechanism controlling PTEN expression via miR-301a in ES cells. Given that PTEN is a pivotal phosphatase factor that regulates cell cycle progression, apoptosis, and proliferation, these results might lead to development of new ES-related therapeutic targets.
\end{abstract}

\section{Introduction}

Ewing's sarcoma (ES) is the second most common primary bone malignancy in children and young adults (1). Patients

Correspondence to: Dr K. Tanaka, Department of Orthopaedic Surgery, Faculty of Medicine, Oita University, Oita 879-5593, Japan E-mail: ktanaka@oita-u.ac.jp

Key words: microRNA, phosphatase and tensin homolog, Ewing's sarcoma with nonmetastatic ES have improved outcomes, however, $30-40 \%$ of recovering ES patients experience recurrence or relapse (2). Traditional therapeutic approaches include local control of the primary lesion by surgery and/or radiation therapy, and treatment of disseminated disease with multiagent cytotoxic chemotherapy. These approaches have led to significant improvements in outcomes in recent decades, particularly in patients with localized disease (3). However, the high percentage of relapse, metastasis, and poor prognosis in ES patients has led to renewed interest in novel treatments.

miRNAs play important roles in cancer development and progression (4). Altered miRNA expression profiles have been reported in various types of cancer, including liver, colon, and prostate $(5,6)$. Functionally, aberrant miRNA expression can affect cell proliferation (7), apoptosis (8), chemo- and radiosensitivities (9), and development of metastases (10). Recent studies indicated that miR-301a is significantly overexpressed in many kinds of cancer, including pancreatic, breast, hepatocellular, and small-cell lung cancers (11-14). However, the role of miR-301a in ES cell proliferation remains unclear.

Phosphatase and tensin homologue (PTEN) is considered as a tumor suppressor gene associated with the p53 signaling pathway $(15,16)$. PTEN plays a vital role in regulating the cell cycle and inhibiting cell growth and division $(17,18)$. Recently, several reports demonstrated PTEN involvement in apoptosis in human hepatocellular carcinoma cells (19), and in cell proliferation, migration, and invasion in gastric cancer (20).

In this study, we analyzed genome-wide microarray expression data for both miRNAs and mRNAs in five human ES cell lines and human mesenchymal stem cells (hMSCs). The results indicated increased miR-301a expression and repressed PTEN expression in all five ES cell lines compared to hMSCs. Based on the inverse correlation observed between miR-301a and PTEN expression, we hypothesized that the effect of PTEN in ES cells might be directly or indirectly mediated in part by miR-301a. Therefore, the purpose of our study was to assess whether PTEN expression was repressed by miR-301a and the role of this pathway in ES cell tumorigenesis.

\section{Materials and methods}

Cell lines. The human ES cell lines, SKES1, RDES, SKNMC and $\mathrm{SCCH}$ were obtained from JCRB Cell Bank (Tokyo, 
Japan), and WE68 was kindly provided Dr Frans van Valen (Westfalische-Wilhelms University, Münster, Germany). Human mesenchymal stem cells (hMSCs) were purchased from Takara Biotechnology (Otsu, Japan). Each line was authenticated as to genotype and phenotype by the source company. RDES and SKNMC cells were cultured in Dulbecco's modified eagle medium (DMEM) high glucose medium (Invitrogen, Grand Island, NY, USA) with 10\% FBS and $1 \%$ penicillin and streptomycin. SKES1 cell were cultured in RPMI-1640 (Invitrogen) supplemented with 10\% FBS. SCCH cells were grown in minimal essential medium (MEM) supplemented with $10 \%$ fetal bovine serum (FBS; Invitrogen) and $0.1 \mathrm{mmol} / 1$ nonessential amino acids (NEAA). hMSC were cultured in mesenchymal stem cell basal medium, chemically defined (MSCBM-CD) with MSCGM-CD SingleQuats (Takara Biotechnology). The cells were maintained at $37^{\circ} \mathrm{C}$ in an incubator with $5 \% \mathrm{CO}_{2}$ and passaged every 2 to 3 days.

Ethics statement. The animal experimental protocol was approved by the Ethics Review Committee for Animal Experimentation of Oita University, and all mice used in this study were anesthetized with ketamine/xylazine or isoflurane/oxygen for experiments and euthanized with cervical dislocation under anesthesia. All efforts were made to minimize suffering.

Mice. BALB/c nu/nu mice (n=28, 6-week old), were acquired from the Kyodo Laboratory (Tosu, Japan). After quarantine, all mice were kept in a pathogen-free environment on a standard 12 h-day/12 h-night cycle and were fed a standard sterilized pellet diet and water ad libitum. All mice were continuously monitored during daytime from Monday to Friday, and twice daily during daytime on Saturdays, Sundays, and holidays for signs of poor health.

RNA isolation. mRNAs were prepared from the triplicate cell cultures using RNeasy kit (Qiagen, Valencia, CA, USA) according to the manufacturer's instruction. The RNA quality was ensured, before labeling, using RNA 6000 Nano kit and Bioanalyzer 2100 (Agilent, Santa Clara, CA, USA). miRNAs were prepared from triplicate cell cultures using the miRNeasy Mini kit (Qiagen) according to the manufacturer's instructions.

Genome-wide miRNA expression microarray. GeneChip miRNA 3.0 array (Affymetrix, Santa Clara, CA, USA) was used for miRNA expression profiling in five ES cell lines and hMSCs. Small RNA $(1 \mu \mathrm{g})$ including miRNA from each sample was labeled with biotin using the FlashTag Biotin HSR kit (Genisphere, Hatfield, PA, USA). Array hybridization, washing and scanning of the slides were carried out according to the manufacturer's recommendations. The data were extracted from the images, quantile-normalized, summarized (median polish), and $\log 2$-transformed with miRNA QC software (Affymetrix). GeneSpring GX 11.0 (Agilent) was used to analyze the array results. Analysis of variance was used to determine the probe sets significantly different between the two groups. The gene list was filtered with a fold-change cutoff of 2 resulting in the output of a list with genes that have significant differential expression at $\geq 2$-fold. Pathway analysis was performed using KEGG pathway Database (http://www.genome.jp/kegg/pathway. html).

Analysis of mRNA expression by cDNA arrays. GeneChip Genome HG U133 Plus 2.0 Array (Affymetrix) was used for mRNA expression profiling in $5 \mathrm{ES}$ cell lines and hMSC. Biotinylated cRNA was synthesized from total RNA using the 3' IVT Express kit (Affymetrix) according to the manufacturer's protocols. In brief, double stranded cDNA was generated by reverse transcription from $1 \mathrm{ng}$ of total RNA an oligo (dT) primer bearing a T7 promoter. The double-strand cDNA was used as a template for in vitro transcription to generate biotin-labeled cRNA. After fragmentation, $12.5 \mu \mathrm{g}$ of cRNA was hybridized to GeneChip array for $16 \mathrm{~h}$. The arrays were washed and stained using GeneChip Fluidics Station 450 (Affymetrix) and then scanned with the GeneChip Scanner 3000 (Affymetrix). The entire experiment was performed twice. Array hybridization, washing, and scanning of the slides were carried out according to the manufacturer's protocols. The microarray numerical values were analyzed using GeneSpring GX 11.0 software: quantile normalization, filter by flags (detected), filter by expression on the normalized data (20.0-100.0th percentile). Analysis of variance was used to determine the probe sets significantly different between the two groups. The gene list was filtered with a fold-change cutoff of 2 , resulting in output of a list with genes with significant differential expression at $\geq 2$-fold.

Target prediction of miRNAs. Basic Local Alignment Search Tool (BLAST), TargetScan 6.0, microRNA.org, was used to search for the predicted target genes of miRNAs.

Prediction of binding site and mature miRNA transfection. Among the predicted target genes of miR-301a in the TargetScan (http://www.targetscan.org/), DIANA (http:// diana.cslab.ece.ntua.gr/microT/), and PicTar (http://pictar. mdc-berlin.de/) databases, we found that PTEN was one of the top candidates. One day prior to the transfection, cells were seeded onto 6 -well plates $\left(1 \times 10^{5}\right.$ cells/well) and incubated with the complete medium without antibiotics $(2 \mathrm{ml} / \mathrm{well})$. Actinomycin D (10 $\mu \mathrm{g} / \mathrm{ml}$, Sigma-Aldrich) was used to inhibit nascent RNA synthesis. The transfection of miR-301a-3p mimic (5'-CAGUGCAAUAGUAUUGUCAAAGC-3') (20 nM), miR-301a-3p mutant (5'-CUCACGUAUAGUAUUG UCAAAGC-3') (20 nM) and negative control (NC) mRNAs (20 nM) (Invitrogen) was performed using Lipofectamine 2000 reagent (Invitrogen) in antibiotics-free OptiMEM (Invitrogen) according to the manufacturer's instructions. After $48 \mathrm{~h}$ of incubation following the transfection, the cells were harvested and processed for further analysis.

RNA extraction, cDNA synthesis, and quantitative real-time $P C R$. Total RNA was extracted from treated with the TRIzol reagent (Invitrogen) and cDNA was synthesized according to the manufacturer's protocol (Roche). Quantitative real-time PCR (qRT-PCR) was performed using a Light Cycler 480 Probe Master System (Roche), and PCR-specific amplification was conducted in the LightCycler ${ }^{\circledR}$ Nano (Roche). The relative expression of genes (PTEN and GAPDH) was calculated with the $2^{-\Delta \Delta \mathrm{Ct}}$ method. The primers used were as listed: (qRT-PCR; 
PTEN-forward 5'-AAGACAAAGCCAACCGATAC-3', PTEN-reverse 5'-GAAGTTGAACTGCTAGCCTC-3'; GAPDH-forward 5'-CCTCTATGCCAACACAGTGC-3', GAPDH-reverse 5'-GTACTCCTGCTTGCTGATCC-3'.

miRNA inhibitor transfection. One day prior to the transfection, SKES1 and RDES were seeded onto 6-well plates $\left(1 \times 10^{5}\right.$ cells/well) and incubated with the complete medium without antibiotics $(2 \mathrm{ml} /$ well). The transfection of hsa-miR-301a inhibitor $(5,10,20,40,80 \mathrm{nM})$ or negative control miRNAs (Control-miR) (5, 10, 20, 40, $80 \mathrm{nM}$ ) (Invitrogen) was performed using Lipofectamine 2000 reagent (Invitrogen) in antibiotics-free OptiMEM (Invitrogen) according to the manufacturer's instructions. After $48 \mathrm{~h}$ of incubation following the transfection, the cells were harvested and processed for further analysis.

Cell proliferation assay. The PTEN expression plasmid (SC119965) was obtained from OriGene Technologies Inc. (IA, USA). The SKES1 and RDES were plated in 6-well plates $\left(1 \times 10^{5}\right.$ cells per well), and were transfected with or without anti-miR-301a inhibitor, negative control miRNA, PTEN plasmid and Mock plasmid vector using Lipofectamine 2000. Then the cells were incubated in antibiotic-free OptiMEM. After $48 \mathrm{~h}$ of the cultivation, the cells were counted using TC10 Automated Cell Counter (Bio-Rad).

Western blotting. Total cellular protein $(15 \mu \mathrm{g})$ was resolved on a precast $10 \%$ Tris-HCl Criterion 10-well gel (Bio-Rad) at $200 \mathrm{~V}$ (300 mAmp) for $30 \mathrm{~min}$. The gel was wet-transferred to a PVDF membrane for $1 \mathrm{~h}$, and blocked with PBST containing 5\% instant dry non-fat milk for $30 \mathrm{~min}$ at room temperature. Primary antibodies (x1000) Rabbit source against PTEN (\#9552), $\beta$-actin (\#4970), Akt (\#4691), p-Akt (\#4060), p21 (\#2947), Bad (\#9292) and p-Bad (\#9291) proteins were obtained from Cell Signal Technology (Tokyo, Japan). Immunocomplexes were visualized with horseradish peroxidase-conjugated antirabbit immunoglobulin G antibodies (x1000) (GE Healthcare, Tokyo, Japan), developed the blots using ECL Prime system (GE Healthcare) with a ChemiDoc camera (ImageQuant LAS 4000mini; GE Healthcare). The quantification of western blot signals was performed by the densitometry with ImageQuant TL software (GE Healthcare). All western blot experiments were repeated at least three times.

Cell cycle analysis. For cell cycle analysis, SKES1 cells were stained with propidium iodide using Cycletest Plus DNA Reagent kit (BD Biosciences) following the manufacturer's protocol, and the cell cycle distribution was analyzed by FACSVerse flow cytometer (BD Biosciences). The percentages of cells in $\mathrm{G} 0 / \mathrm{G} 1, \mathrm{~S}$ and $\mathrm{G} 2 / \mathrm{M}$ phases were counted and compared. The experiments were carried out in triplicate.

Apoptosis assay. The changes in the expression of apoptotic proteins were analyzed by western blot analysis using antibodies against PAR/poly (ADP-ribose) polymerase (PARP) (\#9542) and cleaved PARP (\#9541) (Cell Signaling Technology, Tokyo, Japan) as an index of apoptosis.

The quantification of cell death was determined by fluorescence activated cell sorting (FACS) using Annexin V-FITC apoptosis detection kit (BD Bioscience) according to the manufacturer's instructions. Briefly, $1 \times 10^{6}$ SKES1 cells were seeded and incubated for $24 \mathrm{~h}$, then anti-miR-301a or PTEN expression plasmid was added to the cells followed by incubation for $48 \mathrm{~h}$. The cells were washed with PBS, suspended in Annexin V binding buffer, then added to an Annexin V-FITC solution and propidium iodide (PI) for $20 \mathrm{~min}$ at room temperature. The samples were analyzed by FACSVerse using FACSuite analysis software (BD Bioscience). To verify the ability for induction of apoptosis, SKES1 cells treated with low dose ADM at $5 \mu \mathrm{g} / \mathrm{ml}$ for $24 \mathrm{~h}$ were used.

In vivo tumor-bearing nude mouse models. The experimental metastasis model was established by injection of $2 \times 10^{6}$ cells transfected with anti-miR-301a suspended in $100 \mu \mathrm{l}$ of normal saline into the gluteal region of nude mice (BALB/c nu/nu, Kudo, Tosu, Japan). Three groups were generated: i) untreated control $(n=7)$; ii) transfected with negative control-miRNA $(\mathrm{n}=7)$; iii) transfected with anti-miR-301a $(\mathrm{n}=7)$; and transfected with PTEN expression vector $(\mathrm{n}=7)$. All mice were fed in standard conditions with weight monitoring and sacrificed 6 weeks after the cell inoculation. Tumor size was measured in two perpendicular dimensions parallel with the surface and the depth of the tumor in mice using a caliper. The tumor volume was estimated using the formula $\mathrm{V}=\left(\right.$ Length $\left.\mathrm{x} \mathrm{Width}^{2}\right) / 2$.

Immunofluorescence microscopy. To determine the effect of anti-miR-301a and PTEN expression vector on the protein level of PTEN, we also performed immunofluorescence staining with PTEN (CST) or p-Akt antibodies (CST). After $48 \mathrm{~h}$, the transfected SKES1 cell lines were fixed with $4 \%$ formaldehyde for $20 \mathrm{~min}$, then incubated with $0.5 \%$ Triton X-100. A rabbit anti-Human PTEN/p-Akt antibody was used for immunofluorescence staining. Following three washes with PBS, the cells were incubated with a goat anti-rabbit Alexa Fluor 594 antibody (Life Technology).

Statistical analysis. Statistical analysis was carried out using SPSS 18.0 software (SPSS Japan Inc., Tokyo, Japan). Two-tailed Student's t-test was used for analysis of continuous variables. We determined the differences among more than 3 groups using a nonrepeated measures analysis of variance (ANOVA) and Scheffe test. Results are expressed as the mean \pm standard deviation, and $p<0.01$ was considered to indicate a statistically significant difference.

\section{Results}

Upregulation of miR-301a expression in ES cell lines. The genome-wide miRNA expression profiling using five ES cell lines was carried out to identify miRNAs specifically expressed in ES cells. The array analysis showed that the expression levels of 1054 miRNAs in ES cells were changed (fold-change $>2.0$ ) in comparison with hMSCs (Fig. 1A). Among 1054 miRNAs, 228 were significantly upregulated, whereas 705 were significantly downregulated in all tested ES cells compared to hMSCs. The remaining 121 miRNAs were up- or down-regulated among five ES cell lines. In ES cell lines, the increased expression of miR-301a by 2.63 to 40.21 fold compared with hMSCs was observed. 
A

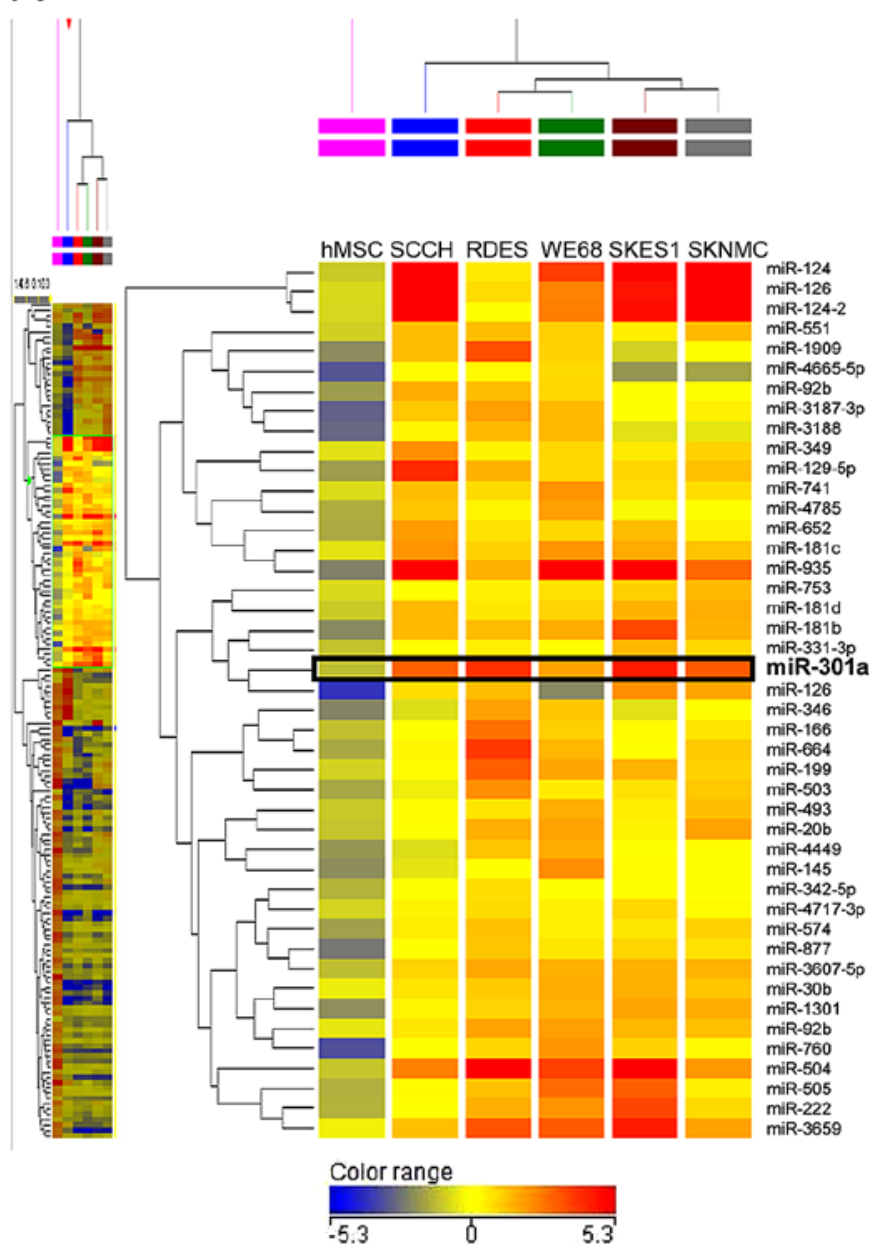

B
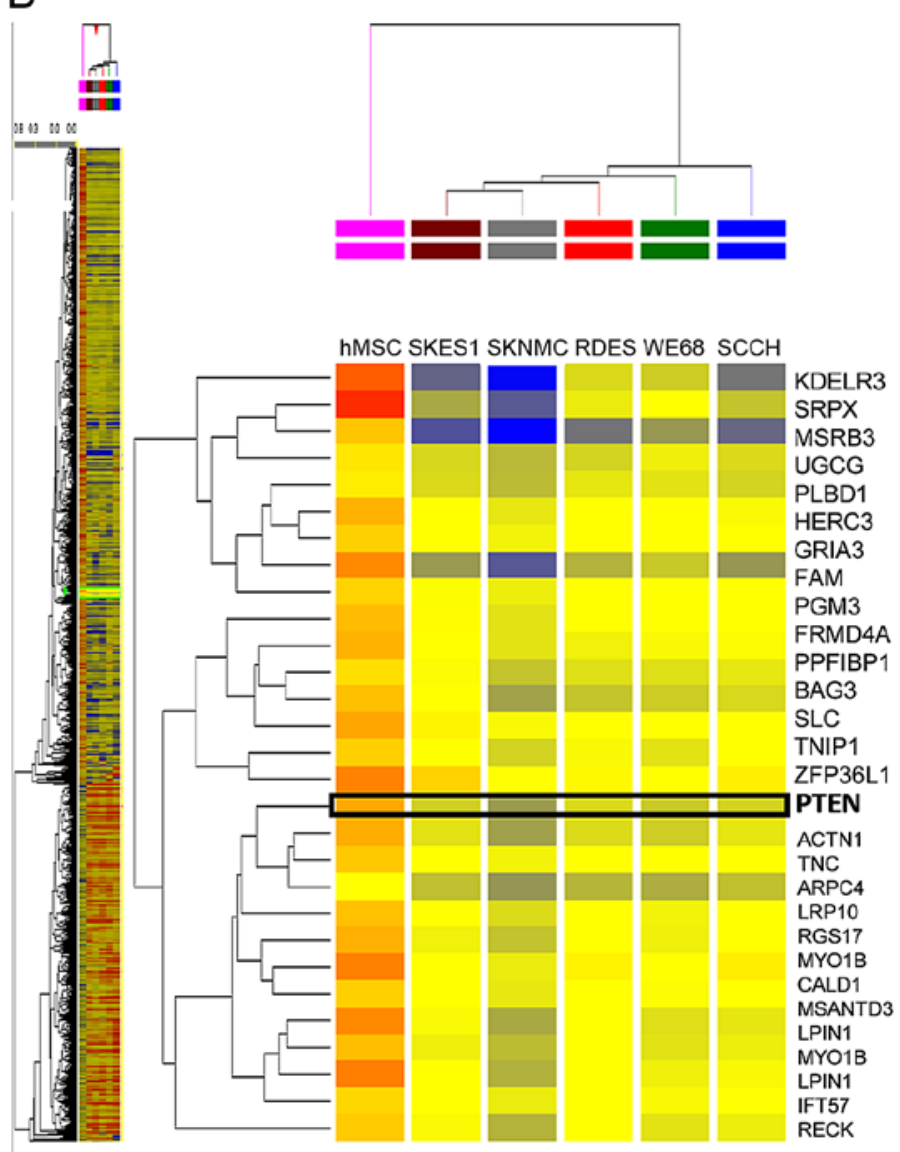

Color range

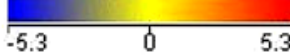

Figure 1. MicroRNA and cDNA microarray analysis of ES cell lines. (A) Heat map of genome-wide miRNA profile in five ES cell lines and hMSCs. miR-301a was downregulated in all five ES cell lines. (B) Whole-genome mRNA profile and extraction of PTEN and related genes indicate involvement in the same pathways in ES cells and hMSCs. The color bar represents the grades of relative expression levels; increases are red, while decreases are blue.

Downregulation of PTEN expression in ES cell lines. The cDNA array analysis demonstrated that the expression levels of 3043 mRNAs were significantly changed between five ES cell lines and hMSCs (Fig. 1B). We found that 1062 genes were significantly upregulated, whereas 1884 genes were significantly downregulated and the remaining 97 genes were up- or down-regulated in five ES cell lines compared to hMSCs. The expression of PTEN was decreased by -2.69 to -6.55 -fold in five ES cell lines.

PTEN as a direct miR-301a target in ES cell lines. The region complementary to the miR-301a seed region was found in the 3'-UTR of human PTEN (Fig. 2A). A considerable complementarity between sequences within the seed regions of miR-301a and sequences in the 3' untranslated region (UTR) of PTEN was predicted, using the algorithms in BLAST and TargetScan. The results suggested that miR-301a might affect the expression of PTEN genes by binding to 3'UTR of PTEN. We blocked de novo mRNA transcription using actinomycin D $(10 \mu \mathrm{g} / \mathrm{ml})$, an inhibitor of mRNA transcription to determine changes in miRNA or mRNA stability. To test whether miR-301a expression affected endogenous PTEN expression, we transfected the miR-301a and miR-301a mutant oligonucleotides, as well as the negative control-miR, into SKES1 cells. We observed an increased miR-301a expression by $7.03 \pm 1.09$-fold compared with miR-93 mutant $(0.99 \pm 0.19)$ or control-miR $(1.00 \pm 0.21)(\mathrm{p}<0.01)$ (Fig. 2B) and decreased PTEN expression at the mRNA level following transfection with the miR-301a-3p oligonucleotide $0.42 \pm 0.26$ compared with miR-301a mutant $(0.76 \pm 0.15)$ or control-miR $(0.79 \pm 0.2)$ $(\mathrm{p}<0.01)$ (Fig. 2C).

Upregulation of PTEN expression by anti-miR-301a and PTEN expression vector. To examine the correlation between miR-301a and PTEN in ES cells, miR-301a inhibitor antimiR-301a was transfected into SKES1 cells. Western blot analysis showed that the expression levels of PTEN markedly increased in anti-miR-301a transfected cells compared with untreated or negative control oligo-transfected cells (Fig. 2D). The protein expression level of PTEN in the anti-miR-301a $(20 \mathrm{nM})$ transfected cells was increased to $3.65 \pm 0.17$-fold of that in the control cells $(\mathrm{p}<0.01)$ (Fig. 2E). To further confirm the effects of PTEN on the growth of ES cells, the transfection with PTEN expression vector was carried out. Although the expression level of PTEN protein in the cells transfected with Mock vector was not significantly affected, that in the 
A

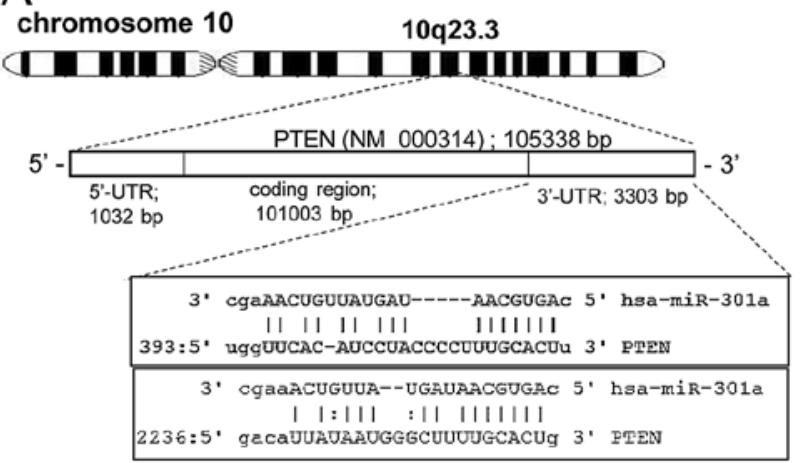

hsa-miR-301a-3p sequence;

WT; 3'- CGAAACUGUUAUGAUAACGUGAC -5'

has-mir-301a-3p mutant sequence;

Mut; 3'- CGAAACUGUUAUGAUUUGCACUC -5'
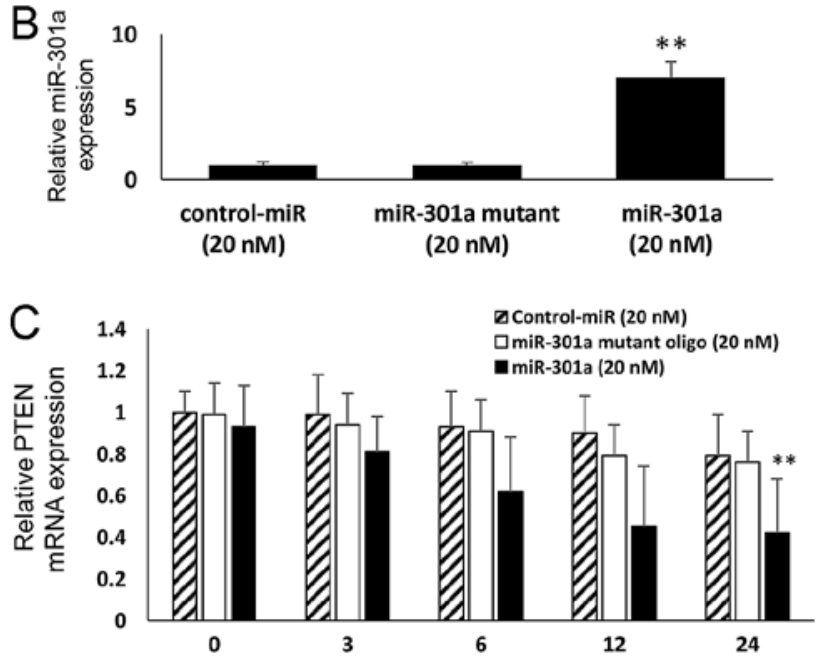

D

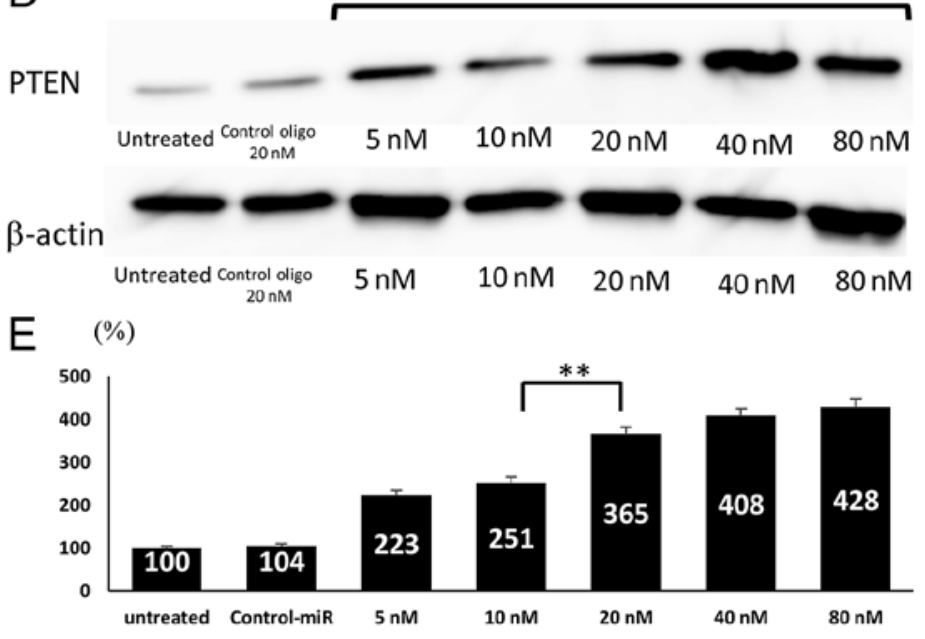

$\mathrm{F}$

PTEN expression vector

PTEN

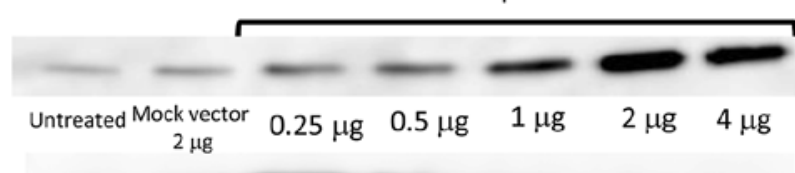

$\beta$-actin

Untreated Mock vector

$2 \mu \mathrm{g}$

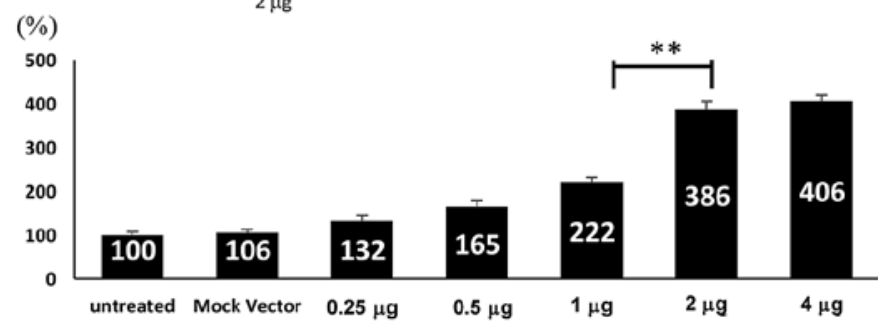

Figure 2. miR-301a inhibition of PTEN mRNA expression in SKES1 tumor cells, and PTEN silencing with anti-miR-301a in ES cells. (A) Predicted miR-301a binding sites at the PTEN mRNA 3'-UTR, as aligned by TargetScan (www.targetscan.org/) and BLAST (http://blast.ncbi.nlm.nih.gov/Blast.cgi). Scheme and sequence of the intact miR-138 (Wt) and its mutant (Mut). (B) After actinomycin D treatment, the expression levels of miR-301a in the negative control-miR, miR-301a, and miR-301a (Mut) were measured by qRT-PCR. (C) After actinomycin D treatment, the mRNA expression levels of PTEN after transfection of negative control-miR, miR-301a, and miR-301a (Mut) was measured by qRT-PCR. (D) PTEN upregulation following anti-miR-301a transfection and PTENexpression vector in ES cells. (E) Densitometry quantification of PTEN protein after transfection of anti-miR-301a. (F) Transfection of PTEN-expression vector in ES cells increased expression of PTEN protein. (G) The quantification of PTEN protein after transfection of PTEN-expression vector. Analysis of variance was carried out to statistically analyze the results. ${ }^{* *} \mathrm{p}<0.01$.

cells transfected with PTEN expression vector was significantly reduced, as determined by western blotting (Fig. $2 \mathrm{~F}$ ). Compared to the control cells (100\%), PTEN vector $(2 \mu \mathrm{g})$ transfected cells exhibited significantly higher PTEN expression level by $3.86 \pm 0.19$-fold ( $<<0.01$ ) (Fig. $2 \mathrm{G}$ ).

Suppression of ES cell growth by transfection of anti301a-miR and PTEN expression vector. PTEN is known to play important roles in the regulation of cell proliferation. Since the transfection of anti-miR-301a resulted in the reduction of PTEN expression, we next examine the effects of anti-miR-301a on the proliferation of ES cells. The cell growth of SKES1 was inhibited by the transfection of anti-miR-301a as determined by cell counting in comparison with negative control-miRNA transfected cells (Fig. 3A). The cell growth of RDES was inhibited by the transfection of anti-miR-301a as determined by cell counting in comparison with negative control-miRNA transfected cells (Fig. 3B). The cell growth of SKES1 was inhibited by the transfection of PTEN expression vector as determined by cell counting in comparison with negative control-miRNA transfected cells (Fig. 3C). The cell growth of RDES was inhibited by the transfection of PTEN expression vector as determined by cell counting in comparison with negative control-miRNA transfected cells (Fig. 3D).

PTEN restoration induced the expression change of Akt. To examine the correlation between PTEN and Akt, the expression of PTEN, Akt, and their downstream factors were investigated in SKES1 cells. SKES1 transfected miR-301a inhibitor and PTEN vector showed increase in expression of PTEN, p21 and phospho (p)-Bad. Western blot analysis showed that the expression levels of p-Akt markedly decreased in antimiR-301a and PTEN vector transfected cells compared with untreated, negative control oligo and Mock vector transfected cells (Fig. 4). 

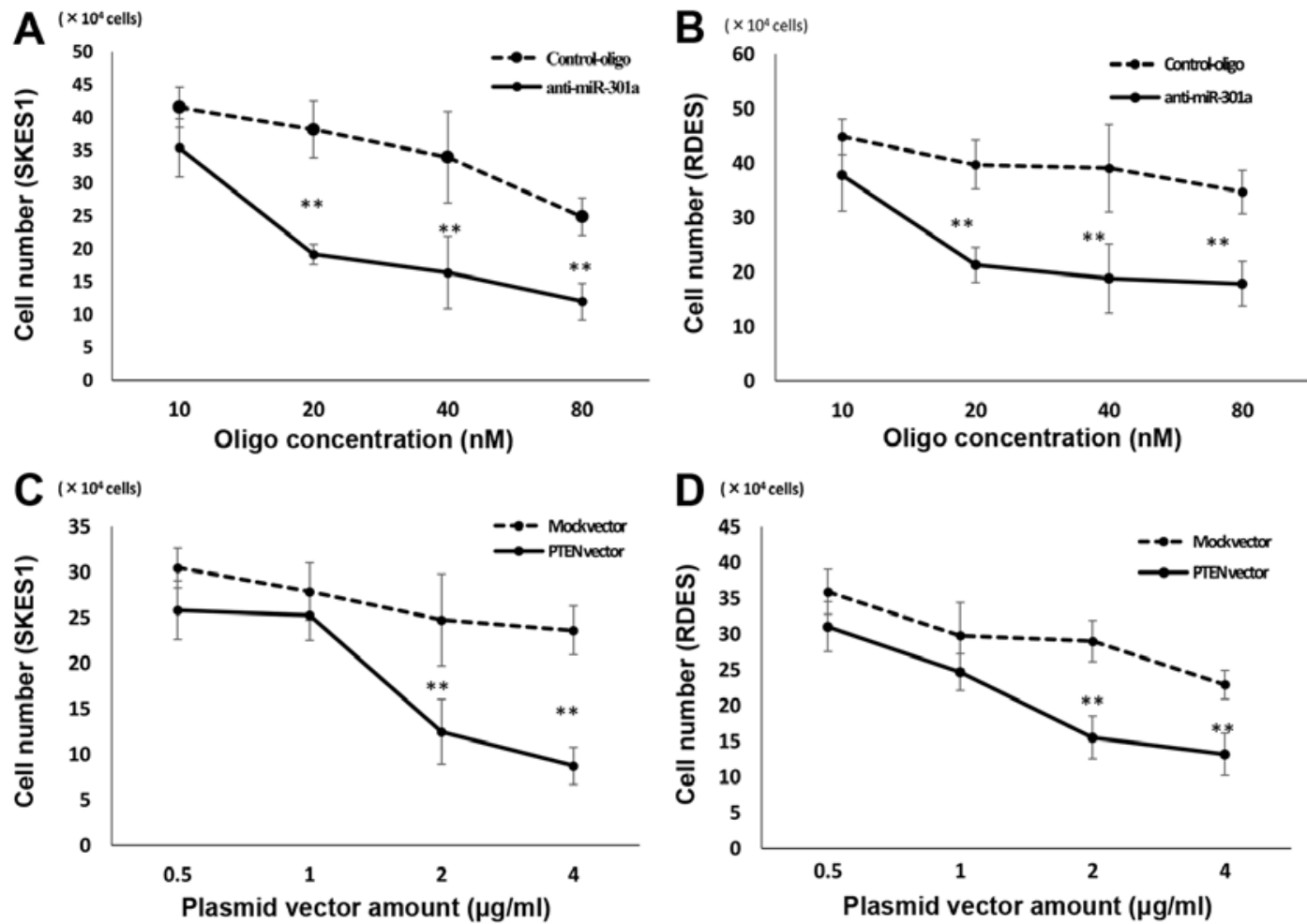

Figure 3. Cell proliferation assay performed to evaluate antiproliferation effects. Cell proliferation assay to evaluate the antiproliferation effects of (A) antimiR-301a and (B) PTEN-expression vector transfection (C and D) into ES cells. Error bars represent mean \pm SD from three independent experiments. The two-tail Student's t-test was employed to statistically analyze the results. ${ }^{* *} \mathrm{p}<0.01$.

Induction of cell cycle arrest at G0/G1 phase by anti-miR301a. Since the introduction of anti-miR-301a significantly inhibited cell proliferation of SKES1 cells, we hypothesized that anti-miR-301a might reduce the cell cycle progression and/or induce-apoptosis of the cells. To monitor the cell cycle distribution, FACS analyses were carried out using antimiR-301a and PTEN plasmid transfected cells (Fig. 5A-D). Both in anti-miR-301a and PTEN plasmid transfected cell lines, the number of the cells in G2/M and G0/G1 phase was significantly lower, and higher than that in the control cells, respectively (Fig. 5E). The data suggested that the repression of miR-301a and restoration of PTEN might have resulted in G0/G1 retardation in ES cells. Then the cellular expression of PARP and its cleaved product was assayed by immunoblotting in SKES1 cells and their transfectants (Fig. 5F). The cleavage of PARP protein, a marker of caspase-mediated apoptosis, was not observed in both anti-miR-301a and PTEN transfectants or the untreated cells and negative control or in mock plasmid vector transfectants. Adriamycin (ADM) is commonly used for the induction of apoptosis as positive control. Additional low dose ADM treatment was able to induce expression of cleaved PARP in both anti-301a-miR and PTEN transfectant, this was not observed in untreated cells, the negative control or the mock plasmid vector transfectants (Fig. 5G). Furthermore, in flow cytometry analysis using Annexin V-FITC/PI double staining, there was no significant difference in the distribution patterns between untreated, negative control miRNA, anti-miR-301a or PTEN plasmid transfected cells (Fig. 5H). Programmed cell death was induced by low dose ADM treatment in anti-miR-301a or PTEN expression vector in SKES1 cells (Fig. 5I).

$$
\begin{aligned}
& \text { Control-miR Anti-miR-301a Mock PTEN } \\
& \text { Untreated }(20 \mathrm{nM}) \quad(20 \mathrm{nM}) \text { vector }(2 \mu \mathrm{g}) \text { vector }(2 \mu \mathrm{g})
\end{aligned}
$$

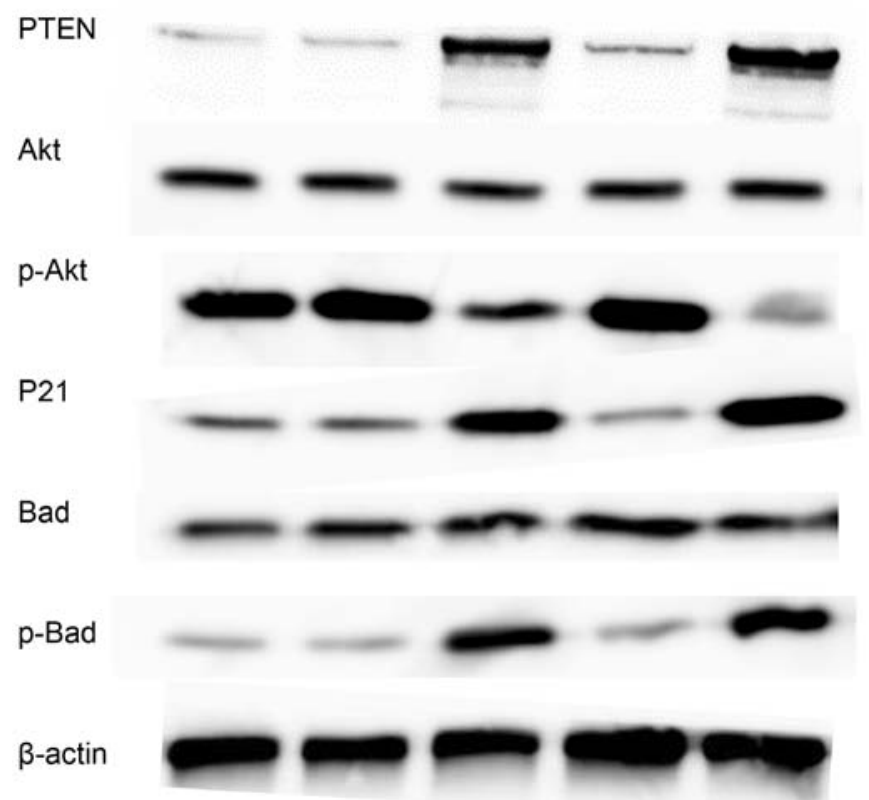

Figure 4. Western blot analysis of PTEN, Akt, and other factors related to the cell cycle and apoptosis. Effect of anti-miR-301a and PTEN-expression vector on PTEN protein expression and its downstream factors in SKES1 tumor cells.

Inhibition of tumor growth in a nude mouse xenograft model by anti-miR-301a. We investigated the efficacy of anti-miR-301a against tumor growth in vivo. The introduction of anti-miR-301a 

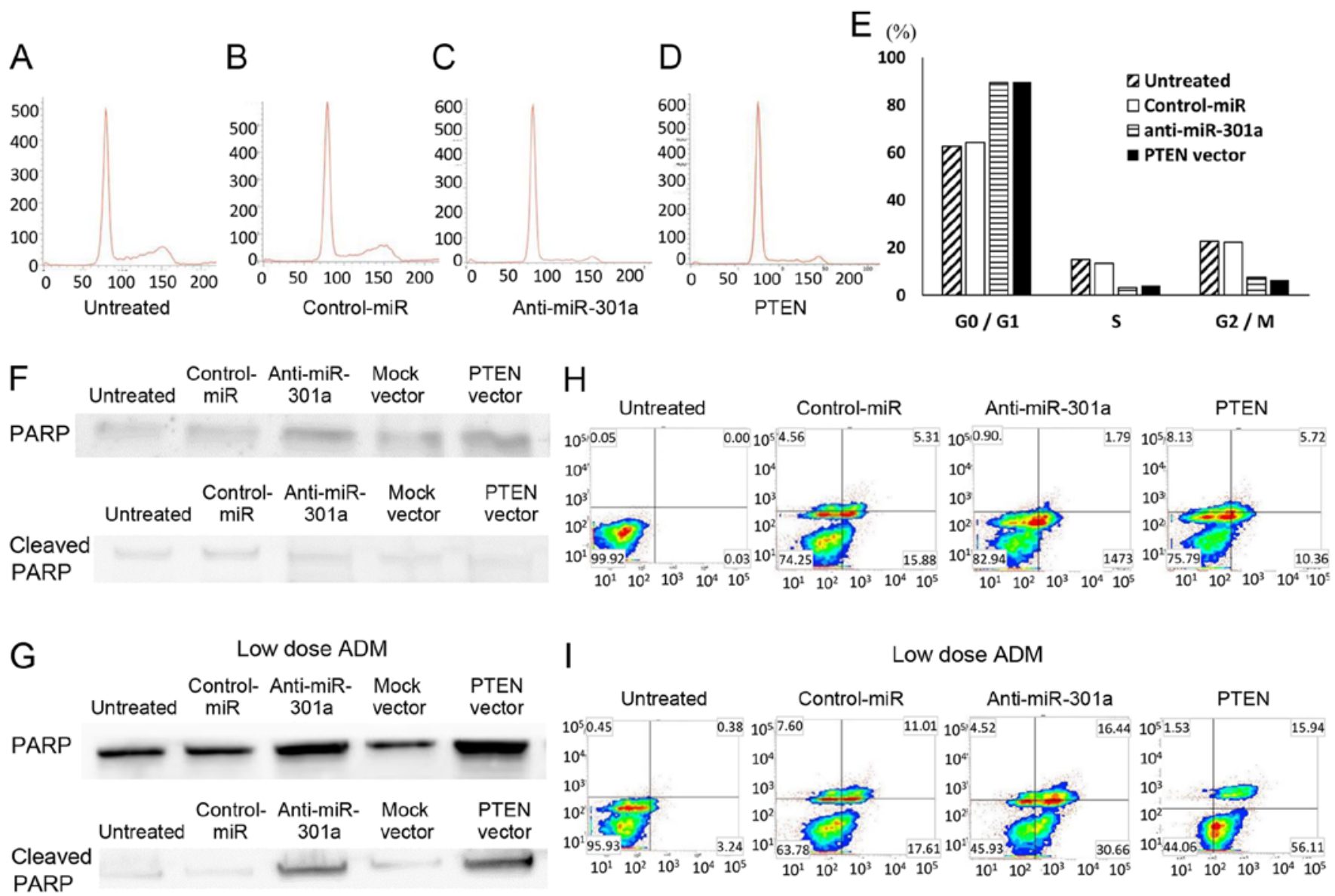

Figure 5. Effects of anti-miR-301a and PTEN-expression vectors on cell cycle progression in SKES1 tumor cells. (A-D) Cells were treated and analyzed by flow cytometry after staining with propidium iodide (PI). (E) Histogram shows quantitative percentage of diploid cells (DNA content) in each cell cycle phase. Effects of anti-miR-301a and PTEN-expression vector transfection on induction of apoptosis in SKES1 tumor cells. (F) Western blot shows the expression of PARP and its cleaved form. (G) Transfection of anti-miR-301a and PTEN-expression vector with low-dose adriamycin showed the expression of PARP and its cleaved form in SKES1 tumor cells. (H) The cells were labelled with fluorescein isothiocyanate Annexin V and PI following oligo-transfection and (I) oligo-transfection with low dose adriamycin. Each quadrant represents viable cells (lower left), early apoptotic cells (lower right), late or secondary necrotic cells (upper right), and primary necrotic cells (upper left).

into SKES1 cells resulted in decreased growth of subcutaneous xenografted tumors in nude mice (Fig. 6A). SKES1 cells transfected with anti-miR-301a $\left(426.7 \pm 107.98 \mathrm{~mm}^{3}\right)$ and PTEN expression vector $\left(355.4 \pm 90.2 \mathrm{~mm}^{3}\right)$ showed statistically smaller tumors in mice compared to untreated $\left(1030.4 \pm 65.4 \mathrm{~mm}^{3}\right)$ and negative control miRNA $\left(927.9 \pm 49.9 \mathrm{~mm}^{3}\right)$ transfected groups (Fig. 6B), indicating that anti-miR-301a also inhibited the growth of ES cells in vivo.

The expression of PTEN (Fig. 6C) and p-AKT (Fig. 6E) within the primary tumor lesion was reduced in the anti-miR301a and PTEN expression vector transfected tumor tissues. The number of PTEN positive cells per unit area was higher $(\mathrm{p}<0.01)$ in the mice with anti-miR-301a $\left(205 \pm 30.8\right.$ cells $\left./ \mathrm{mm}^{2}\right)$ and PTEN expression vector $\left(210.5 \pm 57.9\right.$ cells $\left./ \mathrm{mm}^{2}\right)$ than in those that received untreated $\left(77.7 \pm 16.6 \mathrm{cells} / \mathrm{mm}^{2}\right)$ and control-miR $86.5 \pm 28.1$ cells $/ \mathrm{mm}^{2}$ ) (Fig. 6D). The number of p-Akt positive cells per unit area was lower $(\mathrm{p}<0.01)$ in the mice that anti-miR-301a $\left(127.6 \pm 32.5 \mathrm{cells} / \mathrm{mm}^{2}\right)$ and PTEN expression vector $\left(100.8 \pm 28.1\right.$ cells $\left./ \mathrm{mm}^{2}\right)$ than in those that received untreated $\left(424 \pm 92.2\right.$ cells $\left./ \mathrm{mm}^{2}\right)$ and control-miR (401.2 \pm 127 cells $/ \mathrm{mm}^{2}$ ) (Fig. 6F).

\section{Discussion}

MicroRNAs are small (19-25 nucleotides) noncoding RNAs capable of modulating the expression of their cognate target genes by binding to the 3'-UTR of target mRNAs and causing either translational inhibition or mRNA cleavage (21). Aberrant miRNA expression contributes to the proliferation, invasion, or metastatic behavior of human cancers (22). One miRNA is capable of targeting multiple genes in order to globally regulate a number of biological processes. Moreover, aberrant miRNA expression in human cancer cells causes destruction of miRNA-mediated mRNA networks. Abnormalities in these networks could influence the expression of tumorigenic proteins. To identify important miRNA-mRNA relationships in ES, we performed genome-wide miRNA microarray analysis, as well as cDNA microarray analysis, in ES cells.

Herein, miRNA microarray results demonstrated that the expression of miR-301a was upregulated in all five ES cell lines. Several studies showed that miR-301a was upregulated and involved in various biological and pathological processes, including development, differentiation, inflammation, apoptosis, and cancer metastasis (11-13). The function of miR-301a 


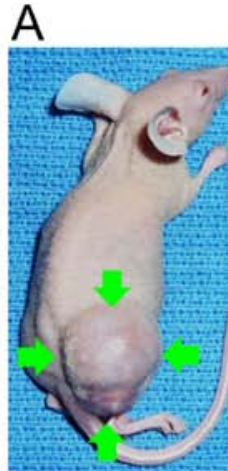

Untreated

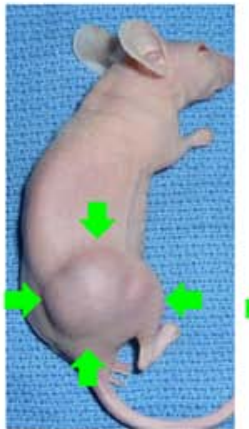

Control-miR

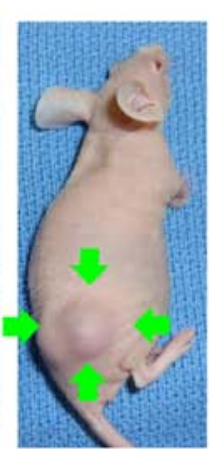

Anti-miR-301a

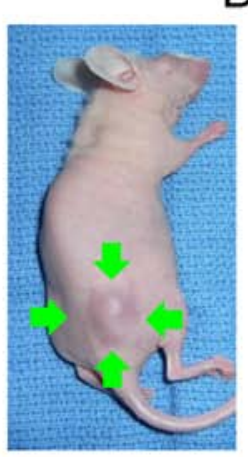

PTEN vector
B $\left(\mathrm{mm}^{3}\right)$

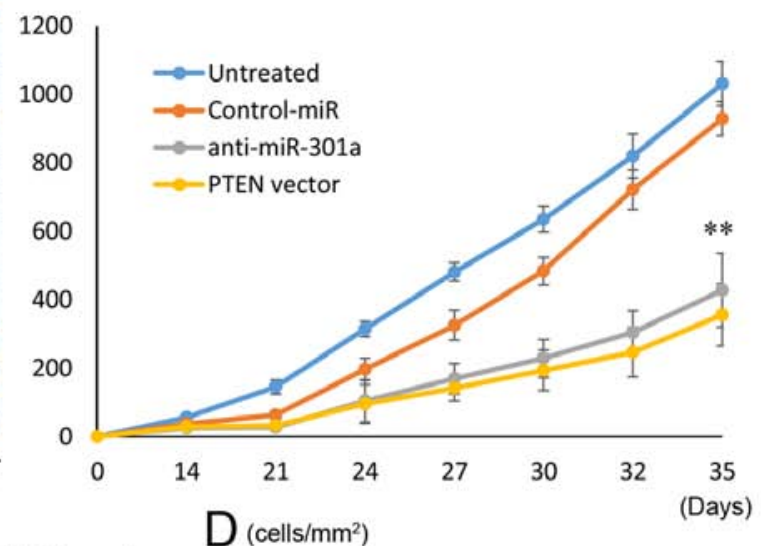

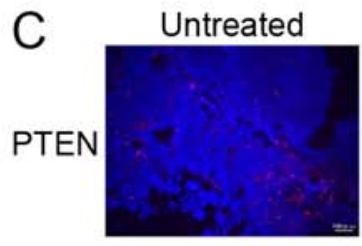
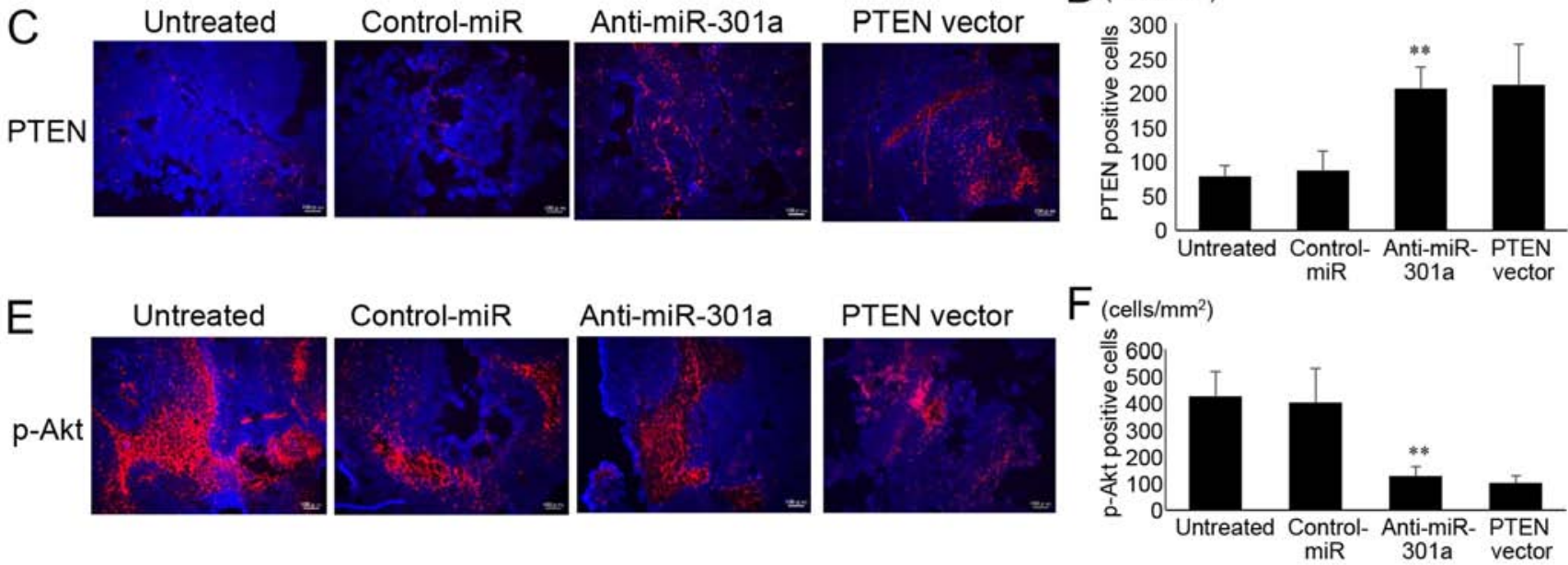

Figure 6. Transfection of anti-miR-301a and PTEN-expression vector suppressed in vivo tumor growth. Four groups: i) untreated (n=7), ii) transfected with of negative control-miRs $(\mathrm{n}=7)$, iii) transfected with anti-miR-301a $(\mathrm{n}=7)$, and iv) transfected with PTEN-expression vector $(\mathrm{n}=7)$. Tumor volumes were measured at the time points indicated after tumor cell inoculation. ${ }^{* *} \mathrm{p}<0.01$. Immunofluorescence of (C) PTEN and (E) p-AKT following transfection of anti-miR-301a and PTEN-expression vector into tumor cells. The number of (D) PTEN- and (F) p-AKT-positive cells per unit area in the tumor lesion.

is important in the ecology of other types of cancer through the targeting of PTEN mRNA (23-25). However, the biological role of miR-301a, including its relationship to PTEN in ES cells, has not been clarified. Our results indicated that the expression of miR-301a was coordinately upregulated in ES cell lines, leading us to investigate genome-wide mRNA profiling by cDNA microarray in order to detect the possible targets of miR-301a in ES cells.

The data from cDNA microarray analysis showed that PTEN mRNA expression was consistently decreased in five ES cell lines as compared with hMSCs. Furthermore, sequence analysis suggested possible association of miR-301a with the 3'-UTR of PTEN mRNA. PTEN is one of the most frequently mutated tumor suppressor genes in human cancers, leading to the activation of the phosphoinositide 3-kinase (PI3K)/ protein kinase B (Akt) signaling pathway and increased cell survival and oncogenesis $(26,27)$. Numerous studies suggested that the transcriptional role of PTEN involves a key position in the complex network of tumor suppressor and oncogenes that regulate cellular transformation. PTEN downregulation was found in lung cancer (28), hepatocellular carcinoma (29), and prostate cancer (30). PTEN level might be related to the biology of Ewing's sarcoma cells and loss of PTEN could result in clinically more aggressive tumors (31).
Our data in ES cells are consistent with previous studies reporting that PTEN downregulation may contribute to malignant potentials (ref. ?).

Although miR-301a might influence the expression of many genes, we focused on PTEN mRNA as a miR-301a target in ES cells. Several genes have been reported as targets of miR-301a, including Bim, RUNX3, Gax and $\operatorname{Nkrf}(11,12,32,33)$. Our cDNA microarray analysis demonstrated that PTEN was the only miR-301a-target gene whose expression was uniformly upregulated in all five ES cell lines, whereas the expression of other candidate genes was different among the ES cells. Analysis using multiple algorithms, such as BLAST (http://blast.ncbi.nlm.nih.gov/Blast. cgi) and TargetScan (www.targetscan.org/), also suggested that PTEN was the putative target of miR-301a. Thus, we analyzed whether miR-301a might contribute to anticancer activities by targeting PTEN mRNA in ES cells. It is possible that miR-301a may have downregulated PTEN expression via indirect pathways. In order to examine whether miR-301a inhibits PTEN mRNA translation, we inhibited PTEN transcription using actinomycin $\mathrm{D}$, and then introduced miR-301a and a miR-301a variant with a mutated seed region. The miR301a mutant did not result in any changes in PTEN levels, while non-mutated miR-301a resulted in reductions in PTEN 
mRNA. Therefore, these results suggested that miR-301a directly inhibited PTEN mRNA function, indicating that miR-301a affects PTEN translation.

We examined the functions of miR-301a in the regulation of its possible target gene, PTEN, and the subsequent changes in the biological characteristics of an ES cell line. The forced expression of miR-301a resulted in the repression of PTEN protein translation, indicating that miR-301a might function as an oncogene in ES cells. Ma et al reported that PTEN, as a cell cycle and proliferation regulator, was the direct target of miR-301a in breast cancer (23). Our results suggested that a similar PTEN regulatory mechanism might exist via miR-301a in ES cells.

Our data regarding the cell cycle showed that miR301a promoted ES cell proliferation by inhibiting cell cycle progression at the G1/G0 phase. These observations were consistent with previous studies demonstrating that PTEN is necessary for the control of the normal G1/S cell cycle checkpoint (34). It was shown that PTEN also played important roles in regulating the $\mathrm{G} 1 / \mathrm{S}$-phase transition and cellular transformation, and that restoring PTEN expression resulted in cell cycle arrest in G1 phase (35). Therefore, we can assume that miR-301a upregulation might affect ES cell cycle progression via miR-301a-mediated control of PTEN expression. It is noteworthy that PTEN upregulation following transfection of anti-301a-miR or a PTEN-expression vector induced apoptosis in ES cells, indicating that the repression of ES cell growth following restoration of PTEN expression implied that PTEN was necessary for cell cycle inhibition, as well as apoptosis induction.

Provided that PTEN acts as a negative regulator of the PI3K/Akt signaling pathway, PTEN repression by miR-301a might play an important role in proliferation of other types of cells (36). Akt activates the mTOR signaling pathway (37) and represses p21, as well as Bax, to enhance cell proliferation preventing apoptosis $(38,39)$. Therefore, Akt downregulation by PTEN in ES cells induced the upregulation of p21 and Bax, which might have resulted in cell cycle inhibition and apoptosis in ES cells.

Furthermore, miR-301a repression resulted in the inhibition of ES tumor growth in vivo. Consistent with the data from in vitro experiments, xenograft ES models also indicated that miR-301a repression was capable of inhibiting ES tumor development in vivo following restoration of PTEN expression and translation.

In summary, our results indicated a novel, inversely correlated relationship between miR-301a and PTEN in ES cells. Our in vitro and in vivo results provided evidence that the expression levels of miR-301a in ES cells were significantly increased and inversely correlated with PTEN expression levels, and that miR-301a played important roles in ES cell proliferation and tumorigenesis by targeting PTEN mRNA. Silenced PTEN has been observed in several human cancers, wherein PTEN inhibition promotes cell growth and proliferation $(40,41)$. Our data suggested that PTEN is one of the crucial factors involved in enhancing tumor proliferation in ES, as well as other malignant tumors. Although confirmation of the results demonstrated in the present study are required using clinical ES samples, these novel findings regarding the link between miR-301a and PTEN in ES cells will be beneficial for understanding ES oncogenesis, and may provide strategies for clinical application.

\section{Acknowledgements}

We would like to thank Katsuhiro Hanada Ph.D, and Takashi Kobayashi, Ph.D, for helpful discussion for this study. This work was supported in part by National Cancer Center Research and Development Fund (26-A-4), the Grants-in-Aid for Scientific Research (no. 24592250) and 15K10451 from Japan Society for the Promotion of Science, and the Grantsin-Aid for Scientific Research from Japan Agency for Medical Research and Development (15Ack0106087h0002).

\section{References}

1. Linet MS, Ries LA, Smith MA, Tarone RE and Devesa SS: Cancer surveillance series: Recent trends in childhood cancer incidence and mortality in the United States. J Natl Cancer Inst 91: 1051-1058, 1999

2. Leavey PJ and Collier AB: Ewing sarcoma: Prognostic criteria, outcomes and future treatment. Expert Rev Anticancer Ther 8: 617-624, 2008.

3. Womer RB, West DC, Krailo MD, Dickman PS, Pawel BR, Grier HE, Marcus K, Sailer S, Healey JH, Dormans JP, et al: Randomized controlled trial of interval-compressed chemotherapy for the treatment of localized Ewing sarcoma: A report from the Children's Oncology Group. J Clin Oncol 30: 4148-4154, 2012.

4. Croce CM: Causes and consequences of microRNA dysregulation in cancer. Nat Rev Genet 10: 704-714, 2009.

5. Motoyama K, Inoue H, Takatsuno Y, Tanaka F, Mimori K, Uetake H, Sugihara K and Mori M: Over- and under-expressed microRNAs in human colorectal cancer. Int J Oncol 34: 1069-1075, 2009.

6. Tong AW, Fulgham P, Jay C, Chen P, Khalil I, Liu S, Senzer N, Eklund AC, Han J and Nemunaitis J: MicroRNA profile analysis of human prostate cancers. Cancer Gene Ther 16: 206-216, 2009.

7. Hiyoshi Y, Kamohara H, Karashima R, Sato N, Imamura Y, Nagai Y, Yoshida N, Toyama E, Hayashi N, Watanabe M, et al: MicroRNA-21 regulates the proliferation and invasion in esophageal squamous cell carcinoma. Clin Cancer Res 15: 1915-1922, 2009.

8. Esquela-Kerscher A and Slack FJ: Oncomirs - microRNAs with a role in cancer. Nat Rev Cancer 6: 259-269, 2006.

9. Ma J, Dong C and Ji C: MicroRNA and drug resistance. Cancer Gene Ther 17: 523-531, 2010.

10. Ma L, Teruya-Feldstein J and Weinberg RA: Tumour invasion and metastasis initiated by microRNA-10b in breast cancer. Nature 449: 682-688, 2007.

11. Zhou P, Jiang W, Wu L, Chang R, Wu K and Wang Z: miR-301a is a candidate oncogene that targets the homeobox gene Gax in human hepatocellular carcinoma. Dig Dis Sci 57: 1171-1180, 2012.

12. Chen Z, Chen LY, Dai HY, Wang P, Gao S and Wang K: miR-301a promotes pancreatic cancer cell proliferation by directly inhibiting Bim expression. J Cell Biochem 113: 3229-3235, 2012.

13. Shi W, Gerster K, Alajez NM, Tsang J, Waldron L, Pintilie M, Hui AB, Sykes J, P'ng C, Miller N, et al: MicroRNA-301 mediates proliferation and invasion in human breast cancer. Cancer Res 71: 2926-2937, 2011.

14. Miko E, Czimmerer Z, Csánky E, Boros G, Buslig J, Dezso B and Scholtz B: Differentially expressed microRNAs in small cell lung cancer. Exp Lung Res 35: 646-664, 2009.

15. Jones N, Bonnet F, Sfar S, Lafitte M, Lafon D, Sierankowski G, Brouste V, Banneau G, Tunon de Lara C, Debled M, et al: Comprehensive analysis of PTEN status in breast carcinomas. Int J Cancer 133: 323-334, 2013.

16. Mayo LD and Donner DB: The PTEN, Mdm2, p53 tumor suppressor-oncoprotein network. Trends Biochem Sci 27: 462-467, 2002.

17. Chu EC and Tarnawski AS: PTEN regulatory functions in tumor suppression and cell biology. Med Sci Monit 10: RA235-RA241, 2004. 
18. Zeng N, Yang KT, Bayan JA, He L, Aggarwal R, Stiles JW, Hou X, Medina V, Abad D, Palian BM, et al: PTEN controls $\beta$-cell regeneration in aged mice by regulating cell cycle inhibitor p16ink4a. Aging Cell 12: 1000-1011, 2013.

19. Li X, Li Z, Li N, Qi J, Fan K, Yin P, Zhao C, Liu Y, Yao W, Cai X, et al: MAGI2 enhances the sensitivity of BEL-7404 human hepatocellular carcinoma cells to staurosporine-induced apoptosis by increasing PTEN stability. Int J Mol Med 32: 439-447, 2013.

20. Yang TS, Yang XH, Wang XD, Wang YL, Zhou B and Song ZS MiR-214 regulate gastric cancer cell proliferation, migration and invasion by targeting PTEN. Cancer Cell Int 13: 68, 2013.

21. Shi Z, Zhang J, Qian X, Han L, Zhang K, Chen L, Liu J, Ren Y, Yang M, Zhang A, et al: AC1MMYR2, an inhibitor of dicer-mediated biogenesis of Oncomir miR-21, reverses epithelial-mesenchymal transition and suppresses tumor growth and progression. Cancer Res 73: 5519-5531, 2013.

22. Nelson KM and Weiss GJ: MicroRNAs and cancer: Past, present, and potential future. Mol Cancer Ther 7: 3655-3660, 2008.

23. Ma F, Zhang J, Zhong L, Wang L, Liu Y, Wang Y, Peng L and Guo B: Upregulated microRNA-301a in breast cancer promotes tumor metastasis by targeting PTEN and activating Wnt//-catenin signaling. Gene 535: 191-197, 2014.

24. Dou L, Wang S, Sui X, Meng X, Shen T, Huang X, Guo J, Fang W, Man Y, Xi J, et al: MiR-301a mediates the effect of IL-6 on the AKT/GSK pathway and hepatic glycogenesis by regulating PTEN expression. Cell Physiol Biochem 35: 1413-1424, 2015.

25. Masliah-Planchon J, Pasmant E, Luscan A, Laurendeau I, Ortonne N, Hivelin M, Varin J, Valeyrie-Allanore L, Dumaine V, Lantieri L, et al: MicroRNAome profiling in benign and malignant neurofibromatosis type 1-associated nerve sheath tumors: Evidences of PTEN pathway alterations in early NF1 tumorigenesis. BMC Genomics 14: 473, 2013.

26. Di Cristofano A, De Acetis M, Koff A, Cordon-Cardo C and Pandolfi PP: Pten and p27KIP1 cooperate in prostate cancer tumor suppression in the mouse. Nat Genet 27: 222-224, 2001.

27. Podsypanina K, Ellenson LH, Nemes A, Gu J, Tamura M Yamada KM, Cordon-Cardo C, Catoretti G, Fisher PE and Parsons R: Mutation of Pten/Mmacl in mice causes neoplasia in multiple organ systems. Proc Natl Acad Sci USA 96: 1563-1568, 1999.

28. Liu ZL, Wang H, Liu J and Wang ZX: MicroRNA-21 (miR-21) expression promotes growth, metastasis, and chemo- or radioresistance in non-small cell lung cancer cells by targeting PTEN. Mol Cell Biochem 372: 35-45, 2013.

29. Jiang J, Zhang Y, Yu C, Li Z, Pan Y and Sun C: MicroRNA-492 expression promotes the progression of hepatic cancer by targeting PTEN. Cancer Cell Int 14: 95, 2014.
30. Kim RJ, Bae E, Hong YK, Hong JY, Kim NK, Ahn HJ, Oh JJ and Park DS: PTEN loss-mediated Akt activation increases the properties of cancer stem-like cell populations in prostate cancer. Oncology 87: 270-279, 2014.

31. Niemeyer BF, Parrish JK, Spoelstra NS, Joyal T, Richer JK and Jedlicka P: Variable expression of PIK3R3 and PTEN in Ewing Sarcoma impacts oncogenic phenotypes. PLoS One 10: e0116895, 2015.

32. Wang M, Li C, Yu B, Su L, Li J, Ju J, Yu Y, Gu Q, Zhu Z and Liu B: Overexpressed miR-301a promotes cell proliferation and invasion by targeting RUNX3 in gastric cancer. J Gastroenterol 48: 1023-1033, 2013.

33. Lu Z, Li Y, Takwi A, Li B, Zhang J, Conklin DJ, Young KH, Martin R and Li Y: miR-301a as an NF- $\kappa$ B activator in pancreatic cancer cells. EMBO J 30: 57-67, 2011

34. Guo SL, Ye H, Teng Y, Wang YL, Yang G, Li XB, Zhang C, Yang X, Yang ZZ and Yang X: Akt-p53-miR-365-cyclin D1/ cdc25A axis contributes to gastric tumorigenesis induced by PTEN deficiency. Nat Commun 4: 2544, 2013.

35. Ou Y, Ma L, Ma L, Huang Z, Zhou W, Zhao C, Zhang B, Song Y, $\mathrm{Yu} \mathrm{C}$ and Zhan Q: Overexpression of cyclin B1 antagonizes chemotherapeutic-induced apoptosis through PTEN/Akt pathway in human esophageal squamous cell carcinoma cells. Cancer Biol Ther 14: 45-55, 2013.

36. Campos M, Kool MM, Daminet S, Ducatelle R, Rutteman G, Kooistra HS, Galac S and Mol JA: Upregulation of the PI3K/Akt pathway in the tumorigenesis of canine thyroid carcinoma. J Vet Intern Med 28: 1814-1823, 2014.

37. Zhang Z, Hou X, Shao C, Li J, Cheng JX, Kuang S, Ahmad N, Ratliff T and Liu X: Plk1 inhibition enhances the efficacy of androgen signaling blockade in castration-resistant prostate cancer. Cancer Res 74: 6635-6647, 2014.

38. Li J, Chen Y, Wan J, Liu X, Yu C and Li W: ABT-263 enhances sorafenib-induced apoptosis associated with Akt activity and the expression of Bax and p21((CIP1/WAF1)) in human cancer cells. Br J Pharmacol 171: 3182-3195, 2014.

39. Vadlakonda L, Pasupuleti M and Pallu R: Role of PI3KAKT-mTOR and Wnt signaling pathways in transition of G1-S phase of cell cycle in cancer cells. Front Oncol 3: 85, 2013.

40. Chen ZX, Sang QT, Du YG and Xin YY: Silence of PTEN in colorectal cancer cells via siRNA inhibits cell growth. J Environ Pathol Toxicol Oncol 33: 233-237, 2014.

41. Nishioka C, Ikezoe T, Yang J and Yokoyama A: Long-term exposure of leukemia cells to multi-targeted tyrosine kinase inhibitor induces activations of AKT, ERK and STAT5 signaling via epigenetic silencing of the PTEN gene. Leukemia 24: 1631-1640, 2010. 